\title{
Präkonzeptionelle Beratung bei Adipositas
}

\author{
Prof. Dr. Volker Briese, Rostock
}

\section{Bei adipösen schwangeren Frauen treten zahlreiche Komplikationen gehäuft auf. Welche Frauen sind besonders gefährdet und was können die betroffenen Frauen schon vor einer geplanten Schwangerschaft tun, um die gesundheitlichen Risiken zu \\ reduzieren?}

Adipositas (Tab. 1) betrifft eine wachsende Anzahl von Menschen. Nationale Studien [1, 2] alarmieren, dass über die Hälfte der Frauen übergewichtig sind. Eine Adipositas (BMI > 97. Perzentile) ist bereits bei $6,3 \%$ der heranwachsenden Kinder und Jugendlichen zu verzeichnen.

Berechnung des Body Mass Index (BMI):

$$
\mathrm{BMI}=\frac{\text { Körpergewicht }(\mathrm{kg})}{(\text { Körperlänge }(\mathrm{m}))^{2}}
$$

Mehr als 1\% der Schwangeren (ca. 7000 - 8000 Schwangere in Deutschland pro Jahr) erscheinen in der Beratung bzw. im Kreißsaal mit einem BMI $>40 \mathrm{~kg} / \mathrm{m}^{2}$; das bedeutet Adipositas 3. Grades.

Ein BMI $>45 \mathrm{~kg} / \mathrm{m}^{2}$ (morbide Adipositas permagna) kommt zu Beginn der
Schwangerschaft in 0,2\% vor. Diese Frauen stellen ein Hochrisikokollektiv dar.

Die Adipositas permagna ist mit Gestationsdiabetes, Präeklampsie, Thromboembolie und kardialen Komplikationen, u. a. Herzinfarkt, assoziiert.

\section{Welche Frauen sind besonders gefährdet?}

Gewicht und Body Mass Index (BMI) allein zeigen das Risiko nicht an. Wichtig ist die Art der Fettverteilung.

Risikopersonen haben hauptsächlich eine zentrale Adipositas. Die Fettansammlungen befinden sich vorwiegend an der Bauchhaut, dem Rücken und den Seiten, sowie intraabdominal. Um die zentrale Form der Adipositas von der

Tab. 1 Gewichtsklassifikation bei Erwachsenen anhand des BMI (nach WHO 2000), Quelle: Deutsche Adipositas Gesellschaft.

\begin{tabular}{|c|c|c|}
\hline Kategorie & BMI & $\begin{array}{l}\text { Risiko für Begleiterkrankungen } \\
\text { des Übergewichts }\end{array}$ \\
\hline Untergewicht & $<18,5$ & niedrig \\
\hline Normalgewicht & $18,5-24,9$ & durchschnittlich \\
\hline Übergewicht & $\geq 25,0$ & \\
\hline $\begin{array}{l}\text { Präadipositas } \\
\text { - Adipositas Grad } 1 \\
\text { - Adipositas Grad } 2 \\
\text { - Adipositas Grad } 3\end{array}$ & $\begin{array}{l}25-29,9 \\
30-34,9 \\
35-39,9 \\
\geq 40\end{array}$ & $\begin{array}{l}\text { Gering erhöht } \\
\text { erhöht } \\
\text { hoch } \\
\text { Sehr hoch }\end{array}$ \\
\hline
\end{tabular}

peripheren abgrenzen zu können, hat man die Waist-to-Hip Ratio (WHR) eingeführt. Dabei wird der Taillenumfang (in $\mathrm{cm}$ ) durch den Hüftumfang (in $\mathrm{cm}$ ) geteilt. Bei Frauen spricht man von abdominaler Adipositas, wenn der Quotient $>0,85$ beträgt.

Das Krankheitsrisiko wird anhand von Laborwerten ermittelt:

- Fettstoffwechsel.

- Leberenzyme.

- Entzündungsparameter (C reaktives Protein, CRP).

- Schilddrüsenfunktion.

- Glukosetoleranz.

- Nüchtern-Insulin.

- Harnsäure.

- Adiponektin (Gewebshormon im Fettgewebe)

Es besteht ein erhöhtes Risiko für Folgeerkrankungen des Herzkreislaufsystems oder für Gestations- und Typ-2-Diabetes, wenn mindestens 3 der folgenden 5 Komponenten des metabolischen Syndroms erfüllt sind:

- Blutdruck über 130/85 mmHg

- Nüchternblutzucker über $110 \mathrm{mg} / \mathrm{dl}$ (6,1 $\mathrm{mmol} / \mathrm{l})$

- Triglyzerid-Werte über $150 \mathrm{mg} / \mathrm{dl}$ $(1,7 \mathrm{mmol} / \mathrm{l})$

- HDL-C-Werte unter $40 \mathrm{mg} / \mathrm{dl}$ $(1,0 \mathrm{mmol} / \mathrm{l})$

- Taillenumfang von mehr als $88 \mathrm{~cm}$

Übergewicht und Adipositas sind häufig mit einer Linksherzvergrößerung asso- 
ziiert. Dieser erworbene Herzfehler kann, unabhängig vom metabolischen Syndrom, im Zusammenhang mit einer Adipositas auftreten.

\section{Gewichtsreduktion vor einer Schwangerschaft}

Die präkonzeptionelle Gewichtsreduktion ist unabdingbar für die Reduktion schwangerschaftsassoziierter Risiken bei übergewichtigen Frauen. Hierbei sind Ernährungsberatung, Anleitung zur körperlichen Aktivität und psychologische Begleitung die wichtigsten Elemente.

\section{Bewegung}

Sport unter Berücksichtigung aller Körperregionen gilt als anerkanntes „Medikament“ zur Reduktion des Bauch- bzw. Taillenumfangs. Täglich 30 Minuten Sport wären optimal.

Aber wie kann das gelingen? Zum Beispiel

- mit einer Taktik der kleinen Schritte

- einem Training in überschaubaren Einheiten

- mit einer Motivation für Wiederholungen durch Erfolgserlebnisse; Motivation ist der stärkste Faktor zum Antrieb.

Das Ausmaß der Gewichtsreduktion ist jedoch kein Maßstab für den therapeuti- schen Erfolg. Anzustreben ist eine „qua-

lifizierte" Gewichtsabnahme:

- Vermeidung von Hunger und Stress;

- Fettdepots ab- und Muskelmasse aufbauen.

\section{Diäten: die Qual der Wahl}

Die Ergebnisse der Nationalen Verzehrsstudie II [2] verweisen auf eine durchschnittliche tägliche Fettzufuhr von 35\% bei Frauen und von 36\% bei Männern bezogen auf die Gesamtenergiezufuhr. Eine Fettzufuhr von $20-30 \%$ ist jedoch ausreichend. Der Verzicht auf überflüssige Kohlenhydrate und Fette, z.B. zuckerhaltige Getränke, konzentrierte Fruchtsäfte, Schokolade und Gebratenes, ist deshalb ein wichtiger Baustein jeder Diät zur Gewichtsreduktion.

- Mit einer energiearmen kalorienreduzierten Diät (<800 kcal/Tag) ist die größte Gewichtsabnahme, minus 15-25\% des Ausgangsgewichts, innerhalb eines kurzen Zeitraumes zu erreichen. Ein langfristiger Erfolg ist jedoch selten.

- Bessere Erfolge bieten die WeightWatchers-Methode mit wöchentlichen Konsultationen sowie

Diätprogramme mit einer Fettreduktion.

- Einige Ernährungsforscher befürworten eine eiweißreiche Kost. Ein zusätzliches Durstgefühl veranlasst den Organismus, mehr Flüssigkeit aufzunehmen. Damit besteht eine zusätzliche sättigende Wirkung.

- Das Optifast-Programm ist ein einjähriges, medizinisch und psychologisch begleitetes Programm zur Gewichtsreduktion. In der Fastenphase besteht die Nahrung aus einer Formula-Diät. Das ambulante Therapieprogramm wird in rund 35 Zentren in Deutschland und Österreich angeboten.

Die Praxis zeigt jedoch: Eine Diättherapie ist hinsichtlich der längerfristigen Gewichtsreduktion nur für einen $\mathrm{BMI}<30$ geeignet.

Präkonzeptionell können mit einer hypokalorischen Diät aber relativ schnelle Gewichtsreduktionen erreicht werden. Gardner et al. [4] haben vier populäre Diäten bei 311 übergewichtigen prämenpausalen Frauen verglichen (Tab.2): Die Atkins-Diät (wenig Kohlenhydrate), die Zone-Diät (ungesättigte Fettsäuren, komplexe Kohlenhydrate), die Ornish-Diät (wenig Fett) und die LEARN-Diät (fettarm und kohlenhydratreich). Sie folgten über ein Jahr einem bestimmten Diätplan, der per TelefonInterview überprüft wurde.

Frauen mit der Atkins-Diät hatten nach zwölf Monaten durchschnittlich 4,7 kg abgenommen; LEARN-Diät $2,6 \mathrm{~kg}$, Ornish-Diät 2,2 kg und Zone-Diät $1,6 \mathrm{~kg}$. Bei der Atkins-Diät war ein Anstieg des HDL-C-Wertes (plus $5 \mathrm{mg} / \mathrm{dl}$ ), eine Sen- 
Tab. 2 Vier Diäten im Vergleich.

\begin{tabular}{|c|c|}
\hline Diät & Besonderheiten \\
\hline Atkins-Diät & $\begin{array}{l}\text { - Wenig Kohlenhydrate, zu Beginn weniger als } 20 \mathrm{~g} \text { pro Tag, } \\
\text { nach } 2 \text { bis } 3 \text { Monaten } 50 \mathrm{~g} \text { täglich (low carb diet) } \\
\text { - Fette ohne Einschränkung }\end{array}$ \\
\hline Ornish-Diät & $\begin{array}{l}\text { - Eingeschränkter Fettkonsum. Nur } 10 \% \text { der aufgenommenen } \\
\text { Kalorien sollten aus Fett stammen. } \\
\text { - Gemüse, Obst und Getreide dürfen in beliebigen Mengen konsu- } \\
\text { miert werden, } \\
\text { - fettarme Milchprodukte nur in Maßen }\end{array}$ \\
\hline Zone-Diät & $\begin{array}{l}\text { - Nahrung besteht zu } 40 \% \text { aus Kohlenhydraten, } \\
\text { - zu } 30 \% \text { aus Fett } \\
\text { - und zu } 30 \% \text { aus Protein }\end{array}$ \\
\hline LEARN-Diät & $\begin{array}{l}\text { - geringer Fettanteil (low fat diet) } \\
\text { - hoher Kohlenhydratanteil }\end{array}$ \\
\hline
\end{tabular}

kung des Triglyzeridwertes (minus $29 \mathrm{mg} / \mathrm{dl}$ ) sowie eine Senkung des diastolischen (minus $4,4 \mathrm{mmHg}$ ) und des systolischen Drucks (minus 7,6 mmHg) zu verzeichnen. Bei den anderen Diäten fielen die Veränderungen geringer aus. Schon innerhalb eines Jahres war eine Abnahme der Compliance zu verzeichnen.

Fazit: Die Frage nach der richtigen Diät ist nicht entscheidend. Liegt die tägliche Kalorienaufnahme unter dem täglichen Verbrauch, kommt es zwangsläufig zur Gewichtsabnahme, d.h., entscheidender als der Fett- oder Kohlenhydrat-Anteil der Nahrung ist die Menge, die verzehrt wird.

\section{Weniger essen in Kombination mit} einem täglichen Sportprogramm ist erfolgreicher als den Fettgehalt auszurechnen.

Die „Zone-Diät“ entspricht am ehesten auch den Ernährungsempfehlungen in der Schwangerschaft. Hierbei ist ein bevorzugter Verzehr von komplexen Kohlenhydraten wichtig, die den Insulinspiegel langsamer steigen lassen (niedriger Glykämie-Index), z.B. Getreideprodukte.

Der Glykämische Index ist ein Maß für die Wirkung des Nahrungsmittels auf nen bei adipösen Frauen.

Internistische Erkrankungen

Störungen des Fettstoffwechsels

Störungen des Eiweißstoffwechsels

(Hyperurikämie/Gicht)

Störungen des Leberstoffwechsels

Dyspnoe, Hyperventilationssyndrom

Tiefe Venenthrombosen den Blutzuckerspiegel. Je höher der Wert, desto höher steigt der Blutzucker.

Zu einer präkonzeptionellen Beratung bei Übergewicht und Adipositas gehört immer ein 75 g oraler Glukosetoleranztest (OGTT).

\section{"Jo-Jo-Effekt“}

Gelingt das Abnehmen, folgt die Stabilisierung der Gewichtsreduktion als eigentliche Herausforderung: Der Jo-JoEffekt steht dem Erfolg zu oft und wiederholt im Wege. Die Balance zwischen der Hunger- und Sättigungsregulation einerseits und noch unbekannten Mechanismen im Gehirn im Sinne eines
Belohnungssystems spielt nun die entscheidende Rolle.

Die Nahrungsaufnahme löst über die Freisetzung von Botenstoffen positive Empfindungen aus. Über Genuss und Lebensfreude wird dann die Gewichtsspirale nach „oben“ beschleunigt. Dabei bewegt sich das "Jo-Jo“ nach oben viel schneller als nach unten.

Eine psychosomatische Unterstützung ist hilfreich, denn die Angst vor einer möglichen Gewichtszunahme kann auch zur Bulimie führen.

\section{Chirurgische Therapie}

„Trotz vieler wissenschaftlich fundierter Behandlungsstrategien, ist der mittelbis langfristige Erfolg konservativer Adipositastherapien unzureichend. Nur 15\% aller Menschen mit Adipositas Grad 1 und Grad 2 ( BMI 30 bis $40 \mathrm{~kg} / \mathrm{m}^{2}$ ) sind in der Lage, substanziell abzunehmen und das erreichte Gewicht über mehr als fünf Jahre zu halten. Eine Adipositas Grad 3 $\left(\mathrm{BMI}=40 \mathrm{~kg} / \mathrm{m}^{2}\right) \mathrm{kann}$ mit konservativen Verfahren nur selten befriedigend therapiert werden“ [4].

Die Indikation zu einer chirurgischen Therapie besteht bei einer Adipositas permagna (BMI >40). Bei vergeblichen konservativen Therapieversuchen und Begleiterkrankungen des metabolischen Syndroms wie Diabetes mellitus sind

Tab. 3 Internistische Erkrankungen und für die Schwangerschaft relevante Komplikatio-

Assoziierte Komplikationen in der Schwangerschaft

Störungen des Kohlenhydratstoffwechsels

Gestationsdiabetes
Präeklampsie, Schlaganfall

Präeklampsie

Fettleberhepatitis

Pulmonale Komplikationen

Thromboembolie 
chirurgische Interventionen bereits bei einem BMI > 35 angezeigt [3]. Mit dem sogenannten „Gastric Bypass“ können stark übergewichtige Menschen bis zu 50\% ihres Gewichtes verlieren. Ein kleiner Restmagen umgeht dabei den Zwölffingerdarm und wird direkt an den Dünndarm angeschlossen.

\section{Adipositas und Risikobewertung}

Tab. 3 zeigt einen Vorschlag zur Risikoabschätzung bezüglich möglicher Schwangerschaftskomplikationen bei Adipositas $\left(\mathrm{BMI}>30 \mathrm{~kg} / \mathrm{m}^{2}\right)$ aus Sicht der Präventivmedizin.

Im Vordergrund der präkonzeptionellen Beratung bei Adipositas stehen

- die Erkennung eines für die Schwangerschaft relevanten Gefährdungspotenzials,

- die Einleitung einer Folsäureprophylaxe,

- der Aufbau von Kondition und Fitness im Zusammenhang mit einer Ernährungsberatung

- und ein internistisches Basisprogramm, welches das kardiovaskuläre Risikoprofil in der Schwangerschaft deutlich vermindert.

\section{Literatur}

1 Robert-Koch-Institut. DEGS: Studie zur Gesundheit Erwachsener in Deutschland, 2012, www.degs-studie.de

2 Max Rubner Institut, Nationale Verzehrsstudie II, 2008, www. mri.bund.de/NationaleVerzehrsstudie

3 Colquitt J, Clegg A, Loveman E, Royle P, Sidhu M, Colquitt J: Surgery for morbid obesity. Cochrane Database Syst Rev 2005; 4: CD003641.

4 Gardner CD, Kiazand A, Alhassan S, Kim S, Stafford RS, Balise RR, Kraemer HC, King AC: Comparison of the Atkins, Zone, Ornish, and LEARN Diets for Change in Weight and Related Risk Factors Among Overweight Premenopausal Women. JAMA 2007; 297: 969-977

5 Hauner H, Bramlage P, Lösch C, Schunkert H, Wasem J, Jöckel KH, Moebus S: Übergewicht, Adipositas und erhöhter Taillenumfang: Regionale Prävalenzunterschiede in der hausärztlichen Versorgung. Dtsch Ärztebl 2008; 105: 827-833

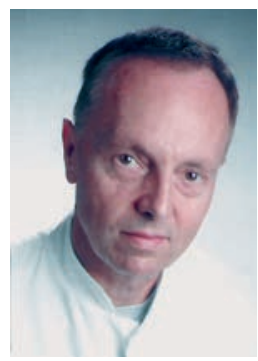

Prof. Dr. Volker Briese

Universitätsfrauenklinik Klinikum Südstadt Südring 81, 18059 Rostock E-Mail: volker.briese@klinikumrostock.de 